\title{
Specially Designed Lattice Structure for Milling Cutter Supported by FEA
}

Pavel Hanzl, Ivana Zetková, Miroslav Zetek

Faculty of Mechanical Engineering, University of West Bohemia. Univerzitní 2732/8, 30614 Pilsen. Czech Republic. E-mail: hanzlp@rti.zcu.cz, zetkova@rti.zcu.cz, mzetek@rti.zcu.cz

Lattice structures are one way to reduce the weight of a component while respecting its strength requirements. These structures are based on cubic cells, therefore, they are not fully applicable to rotating parts which should be lightweight. This article particularly addresses this issue. A solution is sought for how to adapt lattice structures for a milling cutter. The final redesign of the topology allows a continuous flow of generated stress into the whole body of the cutter. Further, the solid part of the milling cutter is modified for Metal Additive Manufacturing (MAM) and the functionality of the optimised cutter is verified by Finite Element Analysis (FEA). The results of the analysis are compared with a conventional cutter with the same outer shell. The findings from the static analysis indicate that the milling cutter can be considered to be competitive.

Keywords: Milling Tool, Topological Optimization, Direct Melting Laser Sintering, Metal Additive Manufacturing

\section{Introduction}

The development of cutting tools is driven forward by the need for efficiency and productivity, and it is a topic which has been addressed by many researchers. The main focus of this research is on material engineering, seeking for ideal process conditions and the geometry of inserts. For example, Botko et al. [1] deal with the influence of different inserts and feed rates on the machined surface quality of aluminium alloy. Other research focuses on material engineering, such as by Zeman et al. [2]. These authors describe the relation between the tool life and the material when milling titanium alloy.

The topic of this paper is unique in regard to a new approach to the production and design of a milling cutter. We carried out topological optimization, which is the process of reducing part weight while respecting strength requirements. Two approaches are distinguished in standard metal additive manufacturing (MAM). One is the bionic approach, where outputs are similar to organic structures like trees or roots. Complex mathematical algorithms are used to construct bionic shapes. The second approach is the use of lattice structures, which are regular porous structures based on a cubic cell. However, the cubic character is not fully applicable for the rotating design of a milling cutter. [3]

There are a large number of possible topologies of lattice structures, but not all are suitable for MAM. If they meet the requirements for manufacturability without support structures, their suitability for the stress conditions of the application is not guaranteed. Selection can be made using a simple rule such as the Maxwell rule [4]. This theme and the benefits of using lattice structures for cutting tools is dealt with in a separate paper [5]. In this paper, BCC topology appears to be very suitable in terms of manufacturability and orientation of its struts with respect to the force load vector.

Significant improvements in the efficiency of selection is achieved by deploying Finite Element Analysis (FEA). However, the search for a suitable topology is always based on the principle of trial and error. [6] A software solution such as OptiStruct provides a comprehensive solution for topological optimization using lattice structures. One of its great advantages is the possibility of creating variable cross-sections of the struts.

Lattice structures in this cutter have a constant strut diameter and this paper solves the issue of how to implement the cubic lattice structures into the rotating body of the cutter. The BCC topology was changed and the directions of the struts was optimized according to the vector of the expected force load and the requirements of MAM.

The entire design process, including basic stiffness verification, is part of this paper. The resulting design was validated by FEA and its manufacturability by MAM was verified. An EOS M 290 printer was used to produce the cutter part. Powdered tool steel 1.2709 was melted using standard process parameters, i.e. a laser power of $285 \mathrm{~W}$ and scanning speed of $960 \mathrm{~mm} / \mathrm{s}$.

\section{Adaptation of Lattice Structure}

In view of the conclusions in [5], the BCC topology was chosen for the input experiments. It meets the manufacturing criteria of MAM with the selected orientation and has 8 struts per node, which according to Maxwell's rule is good for multi-axis stress.

The task is to implement BCC topology into a rotating part. A design into a cluster was thought to be advantageous, so variants of the cutter were divided into clusters. Other variants were also taken into account, but their design resulted in significant changes in topology.

In order to ensure that the border struts are continually linked to the struts of the neighboring cluster, a new topology was designed. The design and count of the clusters was based on the insert number. Because the milling cutter has a cutting diameter of $125 \mathrm{~mm}$ and 6 inserts, each cluster has an angle of $60^{\circ}$. A diagram of the strut distribution in a circular field, which ensures continuous linking, is shown in Figure 1.

The original cubic BCC topology was changed according to this distribution. The $3 \mathrm{D}$ geometry of the basic cell is shown in Figure 2. The occupied volume is $6.3 \mathrm{~mm}$ x $4 \mathrm{~mm} \times 7 \mathrm{~mm}(\mathrm{w} \times \mathrm{dxh}$ ) and the volume fraction was set at $18.7 \%$ and strut diameter is $1.19 \mathrm{~mm}$. The struts grow at $38^{\circ}$, which is sufficient for reliable production of BCC topology. 


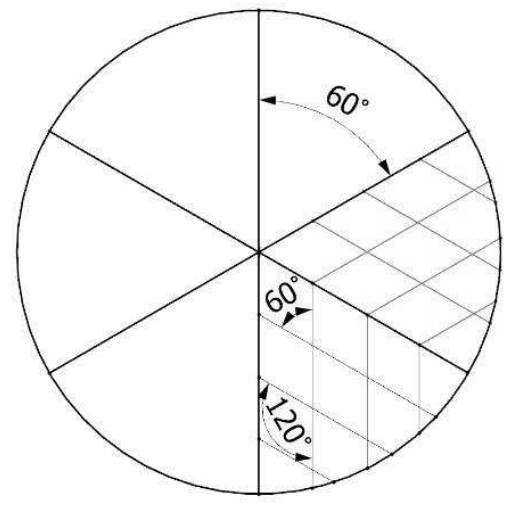

Fig. 1 Schematic cluster distribution in circular field

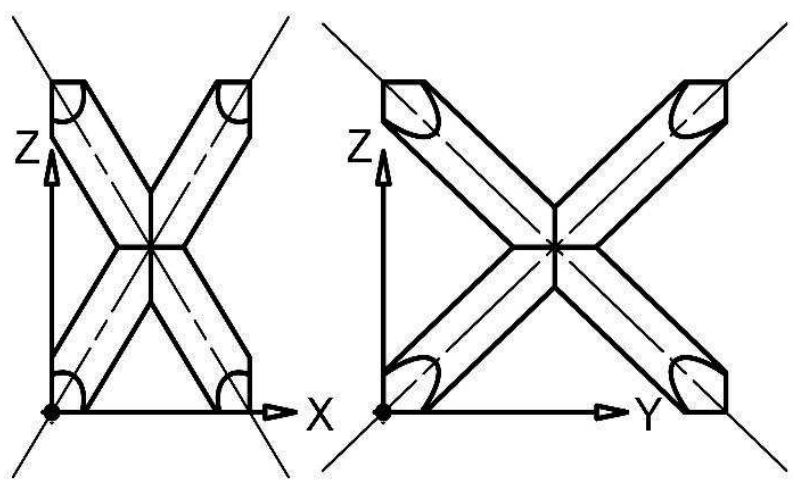

Fig. 2 Geometry of changed BCC topology from cube on the cuboid

This regular lattice structure was inserted into a shell of the cutter body with a thickness of $2.3 \mathrm{~mm}$. The area of the inserts and keyway was strengthened to achieve maximum rigidity. The border of the clusters were positioned before the inserts to ensure maximum absorption of shock generated during machining by the cutter. The interface of the porous structure between clusters is shown in detail in Figure 3.

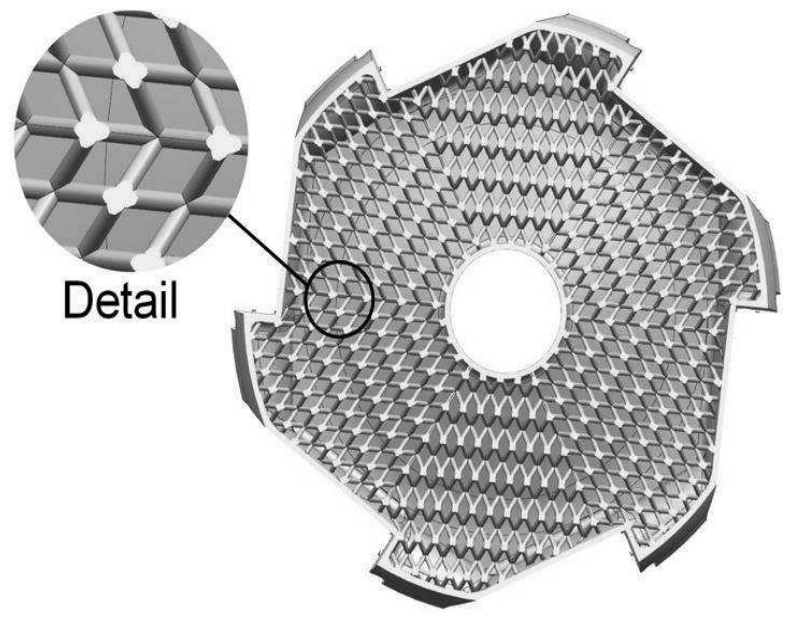

Fig. 3 Schematic cluster distribution in circular field

The shape of the shell body of the cutter was adapted for MAM. A compromise between purpose and manufacturability was sought. The internal surface of the shell is supported by the lattice structures, but the thin angled wall may be problematic to manufacture. However, the structures meet the condition of a building direction higher than $40^{\circ}$. Figure 4 shows a partly built piece. The ellipse indicates the protrusion of melted material built below $45^{\circ}$. The edge of the thin wall began to rise over the current melted layer and the result was a defect of the part accompanied by a collision of the edge with the rigid blade of the recoater. This is caused by a combination of the part position on the building platform, the support structures and the amount of input heat from the laser source. The rising wall of the current layer is a frequent cause of defects when printing thin-walled parts using MAM. The primary area of contact with the recoater and the printed part is particularly critical.

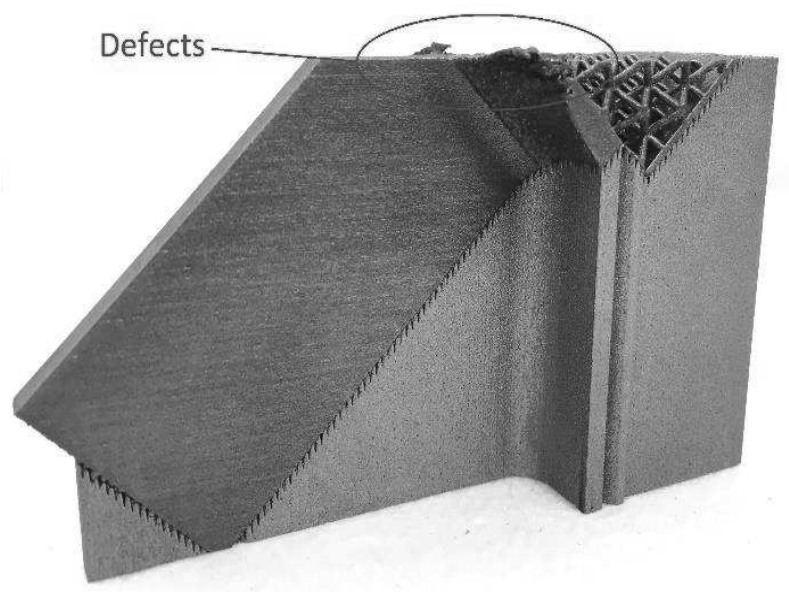

Fig. 4 Defects on the wall in a building direction below $45^{\circ}$

The milling cutter is positioned to ensure its axis is perpendicular to the building platform (along $\mathrm{Z}$ axis), achieving homogeneity of the printed material for each insert. This means that melted layers during MAM have the same normal for each insert bed. Thus, the same characteristics of the insert bed are achieved and possible influences are suppressed. A relatively large impact may have orientations of individual clusters relative to the direction of recoater movement. This effect on mechanical properties cannot be completely avoided and is a production characteristic of MAM. Further, this position of the cutter ensures the minimum height of printed cutter because the angle of the walls can be lower. However, this affects the cost of production. The shape with the technological supports is shown in cross-section in Figure 5.

The whole concept is designed so that its construction is self-supporting and fulfils functionality. The only surface that requires a technological support structure is the bottom surface of the cutter. These structures have the task of supporting the surface with low angles to the platform, anchoring the milling cutter to the building platform and conveying the generated heat during production away from the part to the machine platform. Furthermore, a technological addition of material for the final machining of the surfaces was added. Holes with flat-bottoms were pre-printed to eliminate the deflection of a tiny drill during the production of the threaded holes for the inserts. 


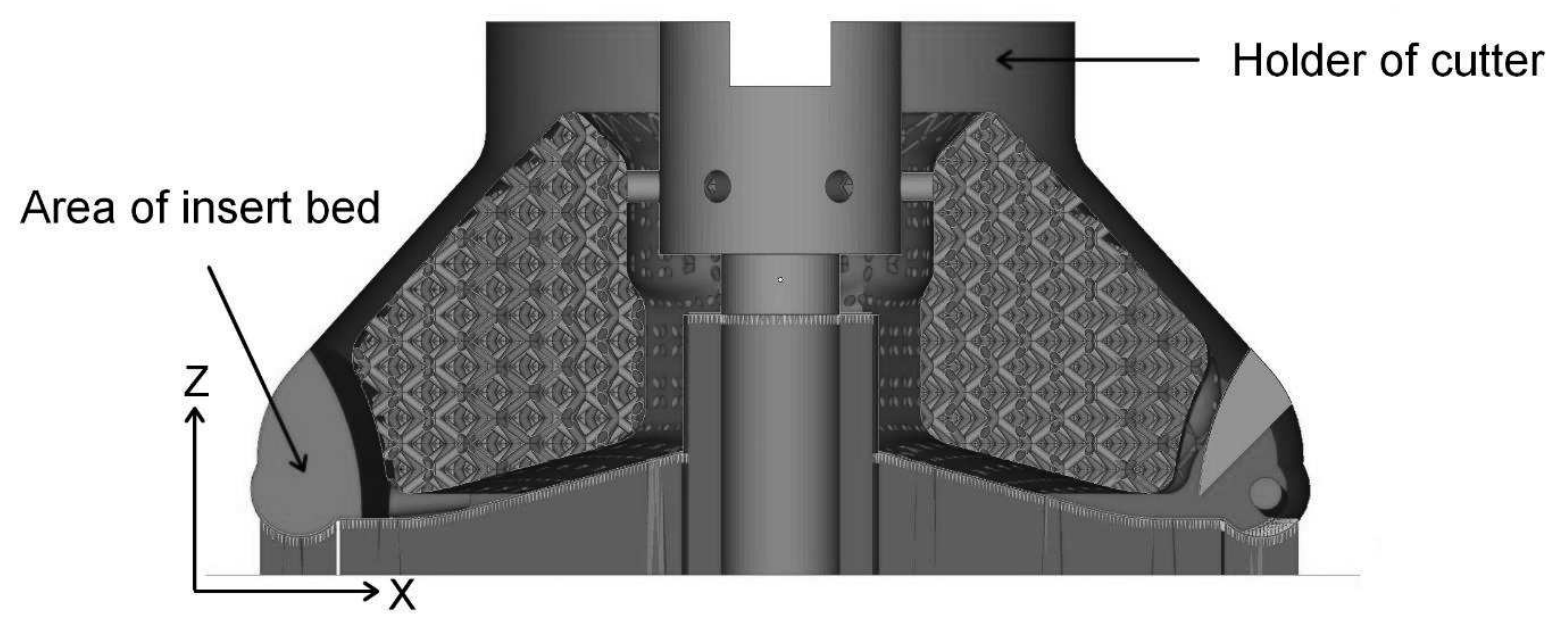

Fig. 5 Geometry of lightweight cutter for MAM; building direction along Z axis

\section{Results and discussion}

Static stress and displacement analyses were carried out. Boundary conditions such as force components were set based on experimental measurements of a solid cutter with the same geometry. Two inserts from six were always under full load during the simulation.

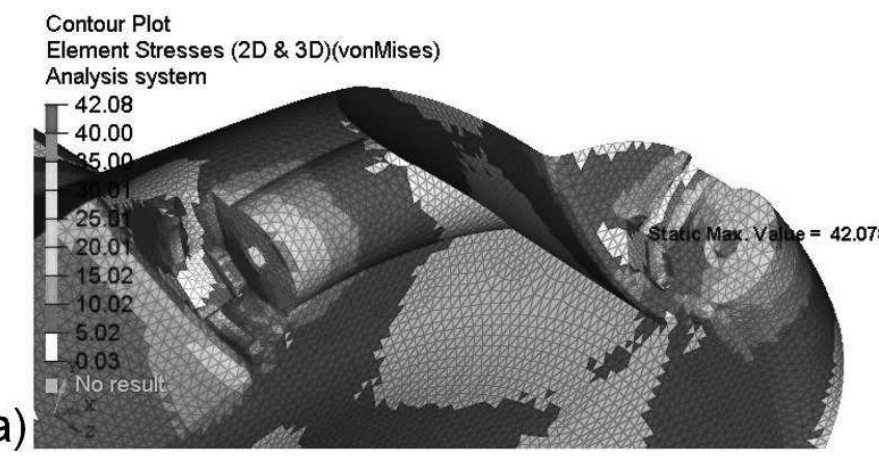

Fig. 6 Stress analysis of lightweight cutter; a) detail of insert bed; b) lattice structure
Analyses proved that the cutter satisfies the strength requirements despite having a lower rigidity than the fully-solid version. There were no problems with production when using the EOS M 290 printer. The finished cutter is shown in Figure 7.

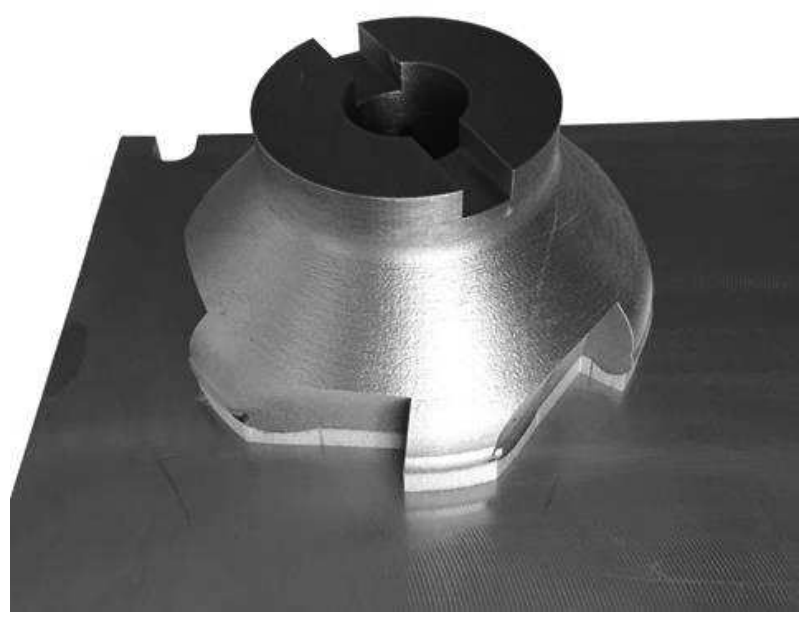

Fig. 7 Printed lightweight cutter without obvious defects
The analyses found that the maximum stress (93.5 $\mathrm{MPa})$ was reached in the area of the holder and the maximum value of the radial displacement of the insert centre was $1.99 \mu \mathrm{m}$. The maxima for the solid cutter were 71.3 $\mathrm{MPa}$ and $0.58 \mu \mathrm{m}$ respectively. The stress fields of the lightweight cutter are shown in Figure 6. These analyses will be described in more detail in a separate paper.

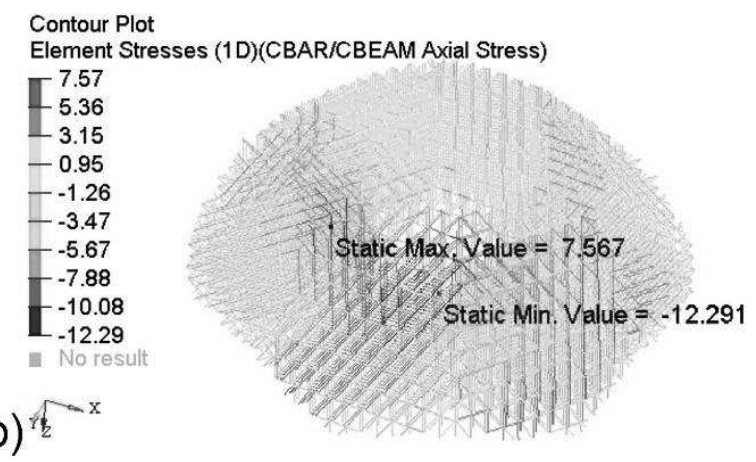

\section{Conclusion}

This paper focuses on increasing the usable potential of a milling cutter. The basic premise is that it must have the ability to withstand cutting forces. Typical approaches to tool rigidity are based on weight gain, which leads to a decrease in tool dynamics, which requires a more powerful spindle drive, which is more expensive and less energetically favourable. Therefore, the tool price is not the only factor in the total costs. A more expensive tool may require a cheaper machine with less spindle power.

Modern approaches try to reduce tool weight and increase efficiency using available materials and design modifications. In professional practice, the process of reducing weight with respect to the functionality of a component is termed 'topological optimization' and one of its methods is to lighten a component by using a lattice structure.

The main aim of this paper was to design a lightweight cutter which combines manufacturability via MAM and static load requirements for milling. The development of a new generation of milling cutter has three components: 
manufacturability, adaptation to stress conditions, and added value of the complete design solution. While this article focuses primarily on manufacturability. If the optimization is done correctly, the resulting product shows an unusual combination of properties, such as high strength and rigidity accompanied by relatively low weight. Good oscillation absorption may be included in this. [3]

A lightweight and firm lattice structure is crucial because it forms the core of the new mill design and affects the resulting behaviour during the cutting process. The BCC cell of the lattice structure was chosen for optimization and its topology was redesigned for the rotating body of the cutter. There are some rules which must be followed to achieve successful production of an optimized milling cutter when using lattice structures.

The cubic character of lattice structures is not fully applicable for the rotating design of a milling cutter. Therefore, the segmentation into clusters was used. In order to ensure transmission between clusters, the size of basic cell of structure is necessary modified. But this solution provides to keep geometry of topology. This is very important for the load capacity of the structure.

In terms of manufacturability, the shell muss be designed for this purpose as well. Angle of its surfaces, which is supported by lattice structures, is sufficient $40^{\circ}$ and the BCC struts were inclined $38^{\circ}$ in this case. This design of the lightweight cutter was checked by FEA and the results did not reveal any areas which would compromise the coherence of the material under the expected loads. However, the analyses was for static loading, and this issue will be described in more detail in a separate paper.

In the final stage, the finished lightweight cutter model was produced by 3D metal printer, EOS M 290. The process of metal additive manufacturing was monitored visually and there were no obvious defects. The body was cut off from the building platform for the final machining of the surfaces. Metal material was added to the outer contour for this process. The holes for the threads were printed to eliminate the deflection of a tiny drill during production of the threaded hole for the inserts.

This paper introduced the design of a topologically optimized milling cutter which is adapted to the requirements of metal additive manufacturing. At the current development stage, the first version of the cutter has been produced and machined. In the following steps, its properties and functionality will be verified by experimental machining. In total, the lightening of the milling cutter has the potential to bring a high degree of innovation.

\section{Acknowledgement}

This paper was supported by the Internal Grant Agency of the University of West Bohemia, project No. SGS2019-008.

\section{References}

[1] BOTKO, F. et al. (2018) Qualitative Evaluation of Machined Surface of Aluminum Alloy AlCu4Mg1 Depend on Feed Rate, Published by Manufacturing Technology, ISSN 1213-2489, April 2018, Vol. 18 , No. 2

[2] ZEMAN, P., BACH, P., TRMAL, G. (2017) Tool Life of PM-HSS Cutting Tools when Milling of Titanium Alloy, Published by Manufacturing Technology, ISSN 1213-2489, February 2017, Vol. 17, No. 1

[3] BENDSOE, M., SIGMUND, O. (2003) Topology Optimization: Theory, Methods and Applications, Springer, ISBN 3-540-42992-1

[4] DESHPANDE, V.S., ASHBY, M.F., FLECK, N.A. (2001) Foam topology: bending versus stretching dominated architectures, Acta Materialia, Volume 49, Issue 6, Pages 1035-1040, ISSN $1359-6454$

[5] HANZL, P., ZETKOVÁ, I. (2019) Benefits of a new approach to designing milling cutter using Metal Additive Manufacturing, Published by Manufacturing Technology, ISSN 1213-2489, April 2019, Vol. 18, No. 2

[6] VERNON R.A. (2016) Discovering optimal unit cell configuration when designing for additive manufacturing using lattice structures, Thesis, Department of Mechanical and Aerospace Engineering, California State University, Long Beach

[7] SYAM, W.P., JIANWEI, W., ZHAO, B., MASKERY, I., ELMADIH, W., LEACH, R. (2018) Design and analysis of strut-based lattice structures for vibration isolation, Published by Precision Engineering, ISSN 0141-6359, Vol. 52, Pp. 494-506. 